\title{
Atracción y retención de recurso humano en salud en zonas rurales: percepción de profesionales
}

\author{
Damaris Meza \\ Universidad de Chile
}

\section{Resumen}

Este artículo busca explorar las motivaciones de profesionales de la salud en zonas rurales. Se busca describir sus percepciones de la permanencia de profesionales para la sostenibilidad de programas de salud rural. Para esto, se realizaron entrevistas semi-estructuradas a médicos, matronas y enfermeras de 5 Centros Comunitarios de Salud Familiar (CECOSF) y 2 Centros de Salud Familiar (CESFAM) de Chiloé, que fueron analizados a través de análisis de contenido narrativo. Se concluye que la permanencia del Recurso Humano en Salud (RHUS) rural es frágil, los factores que inciden en ella son mediados por motivaciones, expectativas y necesidades. Se debe implementar una política de incentivos financieros y no financieros dirigida a médicos y otros profesionales.

Palabras clave: Capital humano en salud, salud rural, análisis de contenido narrativo.

Attracting and retaining human resources in health in rural areas: perception of
professionals

\begin{abstract}
This article seeks to explore the motivations of health professionals in rural areas. It seeks to describe their perceptions of the permanence of professionals for the sustainability of rural health programs. For this, semi-structured interviews were conducted to doctors, midwives and nurses of 5 CECOSF and 2 CESFAM of Chiloé. These interviews were analyzed through analysis of narrative content. It is concluded that the permanence of rural RHUS is fragile, the factors that affect it are mediated by motivations, expectations and needs. A policy of financial and non-financial incentives should be implemented for physicians and other professionals.
\end{abstract}

Keywords: Human capital in health, rural health, analysis of narrative content.

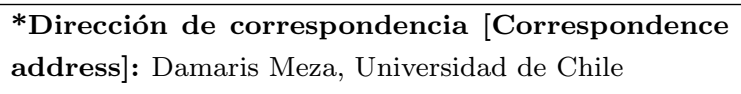




\section{Introducción}

En América Latina y El Caribe la densidad de médicos en zonas urbanas cuadruplica a la del país y en zonas rurales es 8 veces menor. Chile posee un 13,4\% de población rural, sin embargo 219 comunas de 354 en total posee menor capacidad de generación de recursos en el territorio, menor condición socioeconómica y mayores condiciones de ruralidad. En cuanto a la densidad de RHUS el país cuenta con 36 por 10 mil habitantes al año 2013, de esta tasa 24 por 10 mil corresponde al sector público y las comunas rurales tienen una densidad de RHUS de $87,2 \%$ en relación a la densidad de las zonas urbanas.

En este escenario, los países enfrentan un importante desafío en materia de salud pública: garantizar que las personas que viven en zonas rurales y remotas tengan acceso a atención sanitaria oportuna, para ello es indispensable que exista un número suficiente de profesionales de salud competentes en el lugar y el momento adecuados (Organización Mundial de la Salud, 2011). En este sentido, la falta de recurso humano de salud (RHS) en zonas rurales es un problema que afecta a los países a nivel mundial, en especial aquellos en desarrollo.

Al respecto, en los últimos años ha habido un mayor interés por parte de los investigadores y de los responsables de las políticas para identificar y poner en práctica soluciones eficaces para hacer frente a la escasez de personal sanitario en las zonas remotas y rurales. En ese contexto la Organización Mundial de la Salud elaboró recomendaciones para los países, colocando el énfasis en mejorar la retención como estrategia que favorezca el acceso a personal sanitario (Organización Mundial de la Salud, 2011) (Dolea et al., 2010).

Por consiguiente, los países han ideado estrategias para mitigar las brechas de RHS - en mayor o menor medida- pero con importantes falencias en su diseño. Así lo aseguró una revisión sistemática de Dolea y cols. (Dolea et al., 2010), que buscaba evaluar el resultado de las intervenciones para atraer y retener a los trabajadores de salud. Los resultados obtenidos dicen que un número reducido de ellas han tenido evaluación y que el diseño de estas estrategias rara vez se implementan como resultado de un análisis de las preferencias de los trabajadores de salud (Dolea et al., 2010). Esto confirma que urge realizar estudios de preferencias y motivaciones de los profesionales, con el fin de diseñar estrategias coherentes a sus necesidades (Dolea et al., 2010) (Peña et al., 2010).

Chile no está ajeno a esta problemática, los recursos humanos del sector público se han desplazado al privado y concentrado en áreas urbanas, provocando una inequidad en la distribución geográfica de los médicos (Organización Panamericana de la Salud, 2012). El Sistema Nacional de Servicios de Salud cuenta con amplia cobertura en el nivel primario de atención, esto, a través de consultorios urbanos, rurales, CECOSF y postas rurales, sin embargo la permanencia de los profesionales en zonas rurales es menor que en zonas urbanas (Organización Panamericana de la Salud, 2012).

Es por esta razón que esta investigación buscó describir las percepciones de profesionales de salud que se desempeñan en zonas rurales de Chiloé en relación a sus motivaciones para la permanencia en estos territorios y a las políticas de retención de profesionales.

La importancia en salud pública de esta investigación es que conociendo las preferencia del recurso humano las autoridades políticas podrán diseñar estrategias de captación y retención, alcanzar una distribución geográfica más equitativa de profesionales de la salud, mejorar el acceso a la salud de la población rural y, por tanto, mejorar los resultados en salud de la población (Organización Panamericana de la Salud y Organización Mundial de la Salud, 2013).

\section{Metodología}

La investigación corresponde a un estudio de tipo descriptivo-cualitativo. La población estudiada estuvo compuesta por profesionales médicos, matronas y enfermeras que conforman equipos rurales básicos y se llevó a cabo en 5 CECOSF y dos CESFAM en la provincia de Chiloé.

Se realizó un muestreo inicial estratificado en dos grupos: enfermeras, matronas por un lado y médicos por otro, integrantes del equipo de salud de 5 CECOSF de Chiloé, conformando una muestra heterogénea. Se escogieron 5 de los 10 CECOSF existentes en la provincia de Chiloé, que se encuentran emplazados en zonas rurales, aldeas o caseríos, de la isla grande e islas adyacentes. Además, se incluyó en la muestra a profesionales médicos, matronas y enfermeras de otras comunas de Chiloé donde existen CESFAM, comunas consideradas rurales en un 100 por ciento según clasificación INE.

Se aplicaron entrevistas semi-estructuradas a los profesionales antes señalados. El criterio de saturación de información determinó el número de entrevistas por estrato. De acuerdo a los dos grupos conformados se tuvo dos puntos de saturación.

Se utilizó un análisis narrativo de contenido como 
estrategia para interpretar el fondo y el sentido del discurso de los participantes. Se utilizó la estrategia triangulación por investigador con el fin de obtener mayor riqueza interpretativa y analítica.

La investigación se llevó a cabo previa revisión del Comité de Ética de Investigación en seres humanos de la Facultad de Medicina de la Universidad de Chile.

Se entregó una carta de consentimiento informado a los participantes, en donde quedó establecido el carácter voluntario de su participación, la absoluta reserva de sus identidades y el objetivo del estudio. Además, se entregó la transcripción de las entrevistas a cada informante para comprobar la fidelidad de sus discursos.

\section{Resultados}

Los resultados de la investigación son presentados en cuatro ejes temáticos: las motivaciones y necesidades de los profesionales rurales; la sostenibilidad de los programas de salud rural y su relación con la permanencia del RHUS; análisis de políticas actuales de permanencia del RHUS y para finalizar, propuestas de política pertinente de RHUS rural.

\section{Eje motivaciones y necesidades}

\section{Motivaciones y expectativas}

Al indagar sobre las motivaciones presentes en los equipos profesionales rurales, emergen cuatro categorías: las relacionadas con el vínculo familiar tanto directo como lejano con el territorio; aquellas relacionadas con tener la experiencia personal de habitar en la ruralidad; una tercera que incluye las condiciones de vida en una zona rural y por último el acceso a especialización como retribución del trabajo rural.

Estas motivaciones si bien se pueden diferenciar en los discursos de los entrevistados, se presentan en forma interrelacionada en el trascurso de la entrevista, existe una motivación de fondo, la que surge primeramente en las respuestas de los entrevistados y otras motivaciones que se van generando a medida que los entrevistados relatan su experiencia.

En relación al vínculo familiar, se puede señalar que es una de las motivaciones más potentes ya que se relaciona fuertemente con el mayor tiempo de permanencia de los profesionales en la isla. Esto, de acuerdo a lo relatado por los entrevistados que provenían de la isla grande o que estaban ligados a ella por familiares directos, indirectos o amistades que los conectaron al territorio.

Mi motivación principalmente tiene que ver con mi origen chilote, con conocer esta comunidad. O sea, yo siempre he creído que uno que es chilote se contacta de manera muy diferente con las personas a como lo puede hacer... no digo que otra persona no lo pueda hacer, no digo que otra persona que venga de afuera no lo va a hacer bien... pero creo que uno entiende muy bien el lenguaje de la gente de acá, que te puedes conectar muy bien y los puedes ayudar de mucha mejor manera.

(EU, Cesfam insular)

A este respecto se refleja un sentido de pertenencia que se deriva de los discursos, especialmente en aquellos entrevistados que originalmente provienen de Chiloé, estudiaron en otras regiones del país y retornaron a trabajar a su lugar de origen.

En relación a lo anterior, la existencia de redes de apoyo familiar o social en el territorio es un factor que incide en el proceso de adaptación a la ruralidad, se menciona como facilitador para la permanencia. Sin embargo, esta motivación se condiciona por las necesidades insatisfechas del bienestar personal y contractual que se discute más adelante en la categoría de necesidades.

$\mathrm{Y}$ en el mismo aspecto personal, existe un grupo de entrevistados que señala como motivador la experiencia personal de habitar la ruralidad, relatan reflexiones en torno al altruismo de trabajar por personas altamente vulnerables y consideran esta experiencia una aventura en este momento de su vida, pero enriquecedora para el resto de sus vidas.

Respecto a las condiciones laborales y de vida en zonas rurales, para algunos de los entrevistados, la forma de habitar el territorio, la belleza del lugar, la idiosincrasia de las personas, el ritmo de vida y la valoración de la comunidad al trabajo en salud rural se constituye en una motivación relevante. Estas reflexiones están presentes en profesionales que se encuentran en una etapa del ciclo vital más cercana a la adultez media y también en aquellos provenientes de Chiloé. 
A mí me encanta el trabajo en rural, yo encuentro que la gente de campo es gente buena, es gente agradecida, gente de buen trato, (...) es muy agradable trabajar con la gente de acá, además que acá tú haces un verdadero servicio, de verdad tú sirves, porque aquí las condiciones de vida, las condiciones de aislamiento, la dispersión geográfica de nuestra gente hace que tú seas necesaria. ¡Es una maravilla!, Chiloé es bello, Chiloé es grato para vivir, tiene todavía las costumbres provincianas, la gente no anda apurada, hay una calidad de vida 500 veces mejor que en el norte

(Matrona, Cecosf, 57 años)

Además, relevan el hecho de que el quehacer rural sintoniza con sus preferencias al realizar labores diversas, en terreno, en constante movimiento, fuera de la rutina intra-box característico de un trabajo urbano. Esto último se vincula con las expectativas del trabajo rural, que se describe posteriormente en el análisis.

Por otro lado, la motivación específica para médicos relacionada con el acceso a especialización como retribución del trabajo rural sólo está presente en el discurso de los médicos entrevistados, debido a que la política actualmente presente en Chile solo está dirigida a esos profesionales. La estrategia de permanencia de recurso humano en salud que se impone en la ruralidad y que ha permitido el acceso sanitario en las zonas apartadas, es la denominada Etapa de Destinación y Formación (EDF) de médicos generales, por lo tanto, esta motivación resulta relevante para los médicos quienes se encuentra en este ciclo:

Bueno mi principal motivación es el cargo EDF, y lo otro es que el tema de... como del puntaje que tiene mucha relación con la etapa de destinación y formación porque se supone que los lugares rurales tienen cierto puntaje que es mayor y eso te aumenta las perspectivas de tu especialidad después al momento de postular

(médico EDF, 30 años, Cesfam insular)

Junto con lo anterior, los médicos contratados por las corporaciones municipales consideran que trabajar en establecimientos rurales les puede significar una experiencia ventajosa al momento de postular a una beca de especialización en el futuro, beca que se ofrece por la vía de concursos asociados a Periodo de Asistencial Obligatorio (PAO) en un Servicio de Salud del Sistema Nacional de Servicios de Salud, previamente determinado (Ministerio de Salud, 1991):
Y partir por algo público. Siempre...yo no sé... siempre yo lo vi como que la APS es una opción para empezar, pensando en el futuro en poder obtener o que me beneficié el hecho de poder sacar una especialidad

(médico municipal, Cecosf insular)

Un elemento transversal para postular a un cargo son los factores económicos y contractuales, necesarios, pero no suficientes en la atracción. Los informantes exponen que la remuneración les motiva, pero no como único factor, si no ligado a otras motivaciones de índole personal necesarias a la hora de escoger desempeñarse en zonas apartadas.

En el análisis de las motivaciones del equipo rural es preciso profundizar en dos aspectos transversales que potencian la motivación de fondo, actuando en un segundo momento, las que se denominarán motivaciones a la permanencia: trabajo con el equipo e identidad profesional.

La primera tiene que ver con sentirse parte de un equipo rural valorado por la comunidad y que genera lazos afectivos espontáneos entre sí los que se consolidan través del tiempo. Los discursos respecto al soporte que les genera este equipo de trabajo, se explicitan ante la pregunta específica y también surgen en reiteradas ocasiones en el transcurso de las entrevistas. Este aspecto puede generar bienestar o arraigo de los profesionales, pese a las innumerables necesidades a que se ven enfrentados. Más adelante se profundizará en cuáles son esas necesidades.

Aquí surgen relatos de valoración hacia el equipo de salud, por parte de la familia y comunidad, la calidez y cercanía de la gente que vive en sector rural y a partir de eso, tolerar las carencias de recursos (en la categoría necesidades se profundizará en esto)

Yo me siento valorado por la gente de la isla de Lemuy. Siento que los pacientes me quieren, no solamente como médico si no como persona y eso lo valoro y me impulsa a quedarme

(médico EDF, Cesfam insular)

Como segundo elemento, la pertenencia al territorio construye una identidad que los moldea profesionalmente como equipos rurales, generando un compromiso que los valida ante los usuarios hasta el punto de considerarlos un miembro más de la comunidad. 
Yo creo que más por el contacto que se da con la gente, con las personas, es un contacto más íntimo por decirlo así y también para poder ayudar y aportar en el lugar que crecí y que he vivido toda mi vida, porque yo fui, soy de un sector rural y vi esa realidad también y eso fue también lo que quise cuando estudié. Quería trabajar en un sector rural para también ayudar a la comunidad

(EU, Cecosf insular)

Sin embargo, hubo entrevistados que no provenían de Chiloé, los que carecían de sentido de pertenencia, se sentía sin mayor motivación debido al desempeño extremo y algunos de ellos se encontraban a la espera de una nueva oferta laboral menos extrema.

Junto a la exploración de motivaciones, se indagó en las expectativas iniciales que tenían los profesionales respecto del trabajo rural y el nivel de cumplimiento de éstas en el tiempo. Entre ellas se menciona un desempeño rural versátil y tranquilo; el desafío de ejercer como profesional único en un lugar apartado; las condiciones particulares de la población y geografía; la expectativa de temporalidad -aquellos que consideran el cargo como transitorio y otros definitivo; y para finalizar, la expectativa de remuneración.

Mira mis expectativas se han cumplido bastante en el cargo, era más o menos lo que yo esperaba, siempre tenía claro que irse a un sector rural no iba a ser fácil, no iba a estar en un trabajo como de escritorio las 44 horas y tampoco venía con expectativas tan altas, así como para decepcionarme, para nada. O sea, yo sabía a lo que venía, en el fondo.

(Médico EDF, Cesfam insular)

En lo referente a la expectativa del desempeño dinámico dado por la diversidad de actividades que se esperaban desarrollar: asistenciales, comunitarias y administrativas, hay expectativas no cumplidas de acuerdo a la realidad de lo que actualmente hacen. En general están a cargo de múltiples tareas no clínicas, de gestión y administración que se contraponen a la expectativa de un tipo de trabajo diverso pero tranquilo:
Las expectativas era lograr un equilibrio entre el trabajo y la vida personal. Creo que esas expectativas no se han cumplido para nada, o sea, la carga de trabajo con la cual uno se encuentra en un sector rural es mucho más de la que uno espera y... creo que eso también me ha generado bastante desgaste

(médico EDF, Cesfam insular)

La expectativa relacionada a que el profesional sea único en su establecimiento, les significa poner a prueba sus capacidades y conocimientos técnicos, lo que puede considerarse un desafío. Sin embargo, para otros entrevistados no tener un par de la misma profesión, cuando ha transcurrido mucho tiempo (más de dos años aproximadamente), se torna un elemento que produce desmotivación.

Por otro lado, los profesionales tenían una percepción de la geografía de Chiloé menos extrema, sin embargo, las características reales de dispersión (como ejemplo insularidad, distancias, accesos difíciles) estaban lejos de sus expectativas para aquellos que provenían de otras partes del país y entre ellos en especial los que se desempeñan en sectores muy apartados.

Respecto a la expectativa en relación a la temporalidad, se presenta situaciones que van desde una profesional con 10 años de experiencia en establecimiento rural y cuya expectativa inicial era trabajar por un periodo breve para luego estudiar en el extranjero y en contraste una profesional de 57 años que elige Chiloé como lugar para establecerse en los últimos años de actividad laboral.

En lo referente a la remuneración las expectativas no son consensuadas por todos, hay distintas expectativas dependiendo de tres factores que interactúan entre sí: la profesión, la ley bajo la cual están contratados y la antigüedad laboral. Se pueden distinguir tres grupos: el grupo médico con opiniones distintas, en este caso un médico EDF considera que el sueldo no compensa las condiciones de aislamiento y responsabilidades adquiridas y a su vez los médicos municipales, contratados bajo el Estatuto de Atención Primaria (ley 19.873) están conformes con lo remunerado de acuerdo a sus expectativas. Otro grupo conformado por profesionales matronas y enfermeras jóvenes para quienes la pretensión de sueldo se cumple si comparan con un sector urbano, debido a las asignaciones que mejoran el sueldo: asignación de zona, asignación por desempeño difícil y bono trimestral por zona extrema. Por último, a los profesionales mayores de 50 años no se les reconoce su antigüedad para efecto de mayor sueldo, situación que provoca el no cumplimiento de sus expectativas, pero que no les 
afecta su permanencia. Esta situación ocurre debido a factores económicos del empleador o porque los profesionales no cumplen con los requisitos de encasillamiento en la carrera funcionaria, por ejemplo, cuando su experiencia no ha sido exclusivamente en atención primaria.

\begin{abstract}
Ahora, yo sí pensé que iba a ser una vida más relajada, un trabajo más pausado, más parsimonia, pero no ha sido así. Y lo otro también era que uno se espera, bueno, ahora así ha sido, pero yo esperaba que cuando uno llega a un lugar rural, las expectativas de sueldo son más altas, estás más lejos y todo. Y creo que eso tampoco es especialmente atractivo
\end{abstract}

(médico EDF, insular).

Es interesante mencionar la percepción de tres médicos entrevistados que estudiaron medicina en Cuba, quienes relatan expectativas relacionadas con la aplicación del modelo de salud integral, modelo con el cual fueron formados. Y en este aspecto, el cumplimiento de expectativas es mayor, en lo referente a forma de atención, trabajo en terreno, posibilidad de innovación en los sectores con menos población a cargo, aun cuando consideran que la carga administrativa es muy intensa. Por otro lado, ellos perciben que los equipos adolecen de condiciones básicas necesarias para la realización de actividades cotidianas y en ese sentido sí presentan una brecha de expectativas.

$\mathrm{Al}$ indagar sobre los posibles factores que han incidido en el cumplimiento o no de estas expectativas, surge la gestión de la jefatura como condicionante. Mencionan que la jefatura otorga resolución de sus necesidades en forma parcial o tardía, ejemplo de aquello es la falta de insumos mínimos, falta de plan de seguridad en las lanchas de transporte de personal, falta de calefacción, entre otros.

Consideran que hay expectativas no cumplidas que responden a dispersión geográfica o condiciones climáticas, pero la mayor parte de las veces son por problemas de gestión de quien está al mando del establecimiento. Al contrario, cuando existe apoyo del superior jerárquico y actúa como soporte técnico y emocional del equipo, impacta positivamente en el cumplimiento de las expectativas.

\section{Necesidades}

Al analizar los discursos de esta categoría, destaca un factor común en los equipos rurales: las condiciones inadecuadas para su desempeño profesional las que aparecen reiteradamente como necesidad y aspecto de desmotivación. En la categorización, las distintas necesidades referidas por los entrevistados confluyen en tres grupos importantes y comunes: necesidades básicas para el desempeño laboral rural, necesidades de bienestar personal y contractual, necesidades surgidas del aislamiento.

En relación a las necesidades básicas para el desempeño laboral rural, ellos y ellas mencionan: ausencia de calefacción, considerando las bajas temperaturas que se dan en el sur de Chile, falta de box para profesionales, falta de equipos e insumos mínimos para trabajar, movilización insuficiente para realizar trabajo en terreno, ausencia de medidas de seguridad en el desplazamiento a los territorios, entre otras limitantes.

Hemos pasado dos meses sin calefacción (...), entonces eso es muy poco atractivo, ir a trabajar a un lugar donde no tienes calor es muy poco atractivo

(EU, Cecosf)

Los relatos de los profesionales demuestran la falta de recursos materiales mínimos para trabajar. Esta situación hace que muchas veces ellos mismos subvencionen estas necesidades básicas para seguir prestando los servicios, por ejemplo, al comprar combustible para la realización de actividades de terreno o insumos clínicos básicos.

Los equipos insulares mencionan específicamente la necesidad de seguridad en el medio de transporte, especialmente en entrevistados que provienen de otras regiones de Chile quienes les preocupa las condiciones inseguras de traslado y la ausencia de seguro marítimo en caso de accidente.

La utilización de salvavidas no es algo que esté normado, nosotros no usamos salvavidas, están en la lancha. A veces estamos sobrepasados en la cantidad de personas que está permitida para el tipo de navegación, o sea, corremos peligro. Yo creo que eso es lo más...lo que hace que el trabajo no sea perfecto, eso

(matrona Cecosf, insular)

Una segunda categoría reconocible de necesidades es aquellas que afectan el bienestar personal y contractual de los profesionales, especialmente aquellos que deben vivir en los mismos territorios debido al mal acceso geográfico, ejemplo de ello son los equipos profesionales que habitan las islas menores del archipiélago, Apiao y Metahue. Por este 
mismo motivo, el empleador (dependencia municipal) otorga posibilidad de viviendas aledañas al establecimiento rural donde trabajan, como incentivo para los trabajadores, sin embargo, algunos de estos espacios no presentan condiciones mínimas de habitabilidad.

Está la casa-habitación donde supuestamente nos íbamos a quedar; y el año pasado, a los 4 meses de haber empezado a trabajar vino el primer invierno que pasé y la casa se inundó completa. Ya este año llegó el invierno y es lo mismo, es lo mismo. El frío, que no hay leña, que el gas, que el generador no funciona, que no hay agua. [¿En la isla no hay electricidad?] No. Por generador se mantiene el CECOSF. Y así trabajan los otros profesionales que dependen de electricidad como el equipo dental, o la misma calefacción para tenerla encendida a la hora de la ronda para que uno pueda calefaccionarse, o la luz para iluminar algunos box que son oscuros...

(Médico, Cecosf insular)

Evidentemente esta situación genera cuestionamientos en los profesionales, quienes muchas veces ponen en duda su permanencia en el territorio, incluso en aquellos que están más motivados y son originalmente de Chiloé. Sin embargo, ese cuestionamiento no convoca a querer dejar el trabajo rural del todo.

(...) esas son las condiciones que te hacen pensar realmente: ¿sigo en esto, sigo arriesgando mi integridad física o mental por apoyar a la comunidad o me retiro o busco un trabajo donde tenga mejores condiciones que éste? De repente hay días que está malo y tenemos que salir, nos agarró un temporal e igual tenemos que viajar y las condiciones en el mar no son muy favorables cuando está malo el clima

(EU, Cecosf insular)

Aquellos entrevistados que tenían más resueltas sus necesidades básicas de condiciones de vida, relataban la falta de reconocimiento de la jefatura o de quien los empleaba, es decir, no se les demostraba valoración ni se realizaba suficientes acciones de reconocimiento a su rol.

Las necesidades surgidas del aislamiento se manifiestan en: déficit de transporte, problemas de circulación de la información, carencia de comunicación e interacción en los equipos y la necesidad de instancias de esparcimiento y ocio.

Respecto al acceso, los profesionales insulares reclaman un mejor sistema de locomoción inter-islas, que les permita salir o entrar a la isla adyacente, entendiendo que la compleja geografía del Chiloé es estructural y lo que se requiere es intervenir en la movilización colectiva por parte de las autoridades. Esta propuesta de mejoramiento del acceso es mencionada detalladamente más adelante en el eje temático referido a propuestas de política pública.

Otra necesidad que se origina a partir del aislamiento es el problema de circulación de la información que les genera múltiples conflictos especialmente en los CECOSF que dependen de un CESFAM base, en donde la información relacionada con reuniones de programa, capacitaciones o actualizaciones no llegan oportunamente a los equipos rurales.

Eso también implica que tú te aíslas y si de repente hay nueva directiva, nueva normativa del programa y tú no vas a una reunión o a una videoconferencia, ¿cómo vas a saber? Si más encima no tienes internet, menos

(matrona, Cecosf)

Y relacionado con la necesidad de comunicación e interacción, los equipos rurales de los distintos establecimientos no se conocen entre sí, refieren que no existe una instancia de participación de equipos rurales de la provincia de Chiloé que les permita compartir experiencias y hacer redes. Aun cuando no hay relación entre los distintos equipos, suponen que las necesidades en los equipos rurales de Chiloé pueden ser similares, pero no tienen certeza de aquello.

En otra área relativa a la condición de aislamiento, los profesionales entrevistados mencionan que vivir en un sector rural limita el acceso a instancias de esparcimiento y ocio, las que sí se pueden encontrar con mayor facilidad en sectores urbanos, por esa razón algunos han optado por vivir en la ciudad más cercana y desplazarse al sector rural.

(...) pero nunca he pensado en la posibilidad de vivir en Curaco, porque realmente es muy rural, es muy pequeño, no hay posibilidades de que uno pueda salir una noche u otras cosas: supermercados grandes, bencinera... entonces el acceso es muy difícil

(matrona, Cesfam insular)

A continuación, se muestra parte de un relato de una profesional, que, al analizar sus necesidades y el aislamiento como parte de ellas, reflexiona 
en la postergación que sufren las personas que trabajan y viven en un sector rural. Esta sensación de aislamiento y postergación es percibida por los profesionales y la comunidad misma debido a que co-habitan el territorio.

Luego de reflexionar sobre las necesidades presentes se les preguntó si éstas condicionan o no su permanencia en el cargo, y las respuestas tendían a considerar las necesidades como dificultades para ejercer sus funciones, pero no un impedimento para la permanencia. Y vinculando esto a las motivaciones iniciales podemos concluir que estas motivaciones se ven disminuidas por las condiciones adversas que se manifiestan como necesidades insatisfechas para el adecuado desempeño profesional. Sin embargo y pese a la adversidad algunos profesionales presentan tolerancia y adaptación hacia esas condiciones precarias.

Falte lo que falte yo voy a seguir acá, de todas maneras. A mí me gusta caleta esta pega y me gusta el equipo de trabajo que se está formando, entonces para mi es súper cómodo venir a trabajar acá. Me siento súper acogida por la comunidad, como yo trabajo en particular con las mujeres también

(matrona Cecosf, 8 años de servicio)

Es preciso señalar que la vinculación de motivaciones y necesidades actúa distinta dependiendo del grupo que se analice. En el trascurso de las entrevistas y del análisis de los datos, se logra establecer que las motivaciones para los médicos se centran en la especialización médica, tanto por los que están en Etapa de Destinación y Formación, como para aquellos que ven del sector rural, una oportunidad para revalidar su título médico a través del Examen Único de Conocimientos Médicos (EUNACOM) y luego de eso pode optar a una especialidad médica.

A diferencia del grupo de matronas y enfermeras quienes no cuentan con política pública de retención (EDF) y relatan como motivación contar con un vínculo familiar en el territorio y disfrutar de las condiciones de la vida rural, entorno e idiosincrasia. Dentro de este grupo, a las profesionales más jóvenes les motivaba sentirse en un constante desafío al trabajar en sectores rurales.

En cuanto a las expectativas, para el grupo de los médicos, el trabajo en un sector rural se visualizaba como un trabajo versátil, pero tranquilo, situación que no ocurre la mayor parte de las veces debido a la multiplicidad de funciones que tienen que asumir. A diferencia de enfermeras y matronas, quienes esperan tener la práctica rural como experiencia diferente y extrema, junto con poner a prueba sus conocimientos en el caso de profesionales jóvenes. Las profesionales en etapa de adultez media, se establecieron en los territorios con expectativas de permanecer y aportar con su experiencia.

En relación a las necesidades, destaca entre los médicos la falta de equipamiento, infraestructura inadecuada y baja valoración de la jefatura a su desempeño. Para aquellos profesionales (insulares) que cuentan con vivienda otorgada por el empleador manifiestan necesidades importantes de habitabilidad (electricidad, calefacción, filtraciones). Si bien las necesidades son comunes en ambos grupos, las enfermeras y matronas dan cuenta de la dificultad de transporte y son más enfáticas en mencionar la falta de insumos clínicos e insumos generales para la realización de su trabajo. En este sentido, las necesidades de médicos EDF, independiente de lo múltiples que sean, no condicionan su permanencia, ya que su ciclo de destinación los obliga a permanecer unos años, es decir, la vinculación entre necesidades y motivación se da en forma diferente que el resto de profesionales.

\section{Eje Sostenibilidad de programas de salud en la ruralidad}

\section{Particularidades de los servicios rurales}

Las particularidades de los servicios rurales se definen a partir de su diferenciación con los centros urbanos, entre las que se mencionan: acceso a los servicios; tiempo y flexibilidad; cercanía en la relación profesional -con el usuario y con el mundo cultural-; rol social del equipo de salud; y programas rurales.

Para los profesionales entrevistados, la prestación de servicios rurales en comparación al sector urbano se diferencia básicamente en el acceso a los servicios. Cabe señalar que el tema de acceso y aislamiento ya fue abordado anteriormente en el eje temático de las necesidades de los profesionales, pero en este eje son analizadas desde el punto de vista de la prestación de los servicios, en donde el acceso impacta directamente en el quehacer cotidiano de los profesionales. Aquí se puede reconocer las distancias que tiene que recorrer un paciente para llegar al centro de salud, la misma distancia que tiene que sortear el equipo profesional para llegar al domicilio de esa persona, las diferentes vías de acceso a ese domicilio y las condiciones climáticas o mareas que limitan muchas veces el acceso.

El funcionamiento de los establecimientos tiene una dinámica particular, con un sentido del tiempo y flexibilidad distinto a la atención de salud de 
un establecimiento urbano. Por ejemplo, el manejo de agendas para la citación de los pacientes, en un sector rural la agenda médica y profesional es más flexible, debido a los distintos factores de dispersión o clima, por lo que la atención en ronda médica o en CECOSF es más probable que se atrase. Asimismo, los profesionales son también menos estrictos con los atrasos o ausencias de los usuarios, basado en el conocimiento de la realidad local y la vivencia rural compartida, lo que genera empatía y entendimiento mutuo.

La flexibilidad también se manifiesta en que los usuarios y usuarias respetan el tiempo de espera para su atención, asimismo el equipo de salud se tiene que adaptar a la posibilidad de acceso de los usuarios de manera de resolver los problemas de salud de las personas.

Este diálogo entre profesional y usuario genera entendimiento mutuo y da lugar a esta categoría que diferencia a los servicios rurales, cercanía de la relación profesional-paciente. Esta proximidad se da por el conocimiento de la realidad que viven las personas de la comunidad y un equipo próximo que empatiza con el usuario y su familia.

Sabemos dónde vive, sabemos lo que les cuesta llegar, sabemos el drama que tenemos en la casa y que por eso no llegó, no porque se haya quedado mirando novelas. Es otra mirada, porque se conoce a la gente que viene, entonces se le dan muchas facilidades, se hace mucho más humana la atención, encuentro yo, que en otros lados

(matrona, Cecosf)

La cercanía en la atención también se establece debido al conocimiento y valoración de los profesionales hacia los elementos culturales presentes en el territorio. La realidad geográfica e histórica detrás de un paciente establece sus comportamientos en salud, y cuyo entendimiento determina la atención pertinente y cercana del equipo de salud.

Creo que además hay programas como el cardiovascular que en nuestro sector rural o en Chiloé en general hay un tema cultural con la alimentación que es súper difícil de trabajar. La alimentación de las personas en el sector rural es alta en calorías, en grasa, etc. Entonces llegar a decirles que no tienen que comer esas cosas es complicado porque la gente no te hace caso, entonces es un trabajo permanente de estar repitiendo cuales son las indicaciones, considero que a la nutricionista ahí le tocó una tarea bien complicada

(matrona, Cesfam insular)
Es interesante cómo el profesional hace alusión a características de la idiosincrasia chilota para ejemplificar el tipo de dinámica de funcionamiento y de diálogo entre profesional y paciente, así como los aprendizajes que ellos obtienen con la riqueza cultural de Chiloé.

Yo creo que la paciencia es fundamental dentro de los equipos, porque nuestra población tiene paciencia si han vivido toda su vida acá en el sector rural donde saben que, si el bus no pasó, no se va a acabar el mundo y muchas veces el equipo reaccionamos, si no está el examen el mundo se acaba

(médico EDF, Cesfam insular)

Por último, el elemento diferenciador de una atención rural de una urbana es el rol social que muchas veces les toca asumir a los equipos rurales en sus comunidades, ya que son considerados actores relevantes para la comunidad:

También relatan que son consultados de temas no directamente relacionados con prestaciones a salud, pero que sin duda afectan el bienestar individual y colectivo de sus habitantes, en donde podemos aludir al enfoque de determinantes sociales en salud como perspectiva, presente en la concepción integral de la comunidad.

Hay cosas no resueltas que se le demandan a salud que uno no tiene nada que ver, no tienes como gestionar. Por ejemplo, acá un alcantarillado que nunca se realizó, la gente le frustró mucho, para ellos el alcantarillado es un tema de salud, pero no es una resolutividad que vaya por salud en cuanto a plata, es una cuestión de "obras" entonces ahí a nosotros nos ha tocado respirar, aprender, entrar a mover redes con alcaldes, concejales, todo y no... sin querer queriendo hemos podido ir ayudando a solucionar el problema, pero nos ha costado mucho

(matrona, Cecosf)

Por otro lado, como elemento de contexto, es necesario señalar que los programas de salud que se llevan a cabo en los centros de salud rural son los programas ministeriales que se aplican en cualquier centro de salud urbano, con algunas particularidades que se describirán más adelante en el análisis. En otras palabras, un establecimiento de salud rural posee un número importante de prestaciones asociadas a los programas de salud, sin embargo, realiza sólo aquellas que no requieren mayor tecnología ni equipamiento ya que las más complejas 
son resueltas en el centro asistencial cabecera de la comuna (CESFAM).

\section{Profesional integrando la comunidad}

Esta categoría se vincula íntimamente a la cercanía en la relación profesional- paciente y hacia el mundo cultural, entendido como un proceso respecto a la forma de trabajo en sector rural. Se profundizó en cómo el profesional se conecta con la comunidad, cómo se inserta en ella luego de ingresar al trabajo rural. Es importante aclarar que las distintas estrategias mencionadas por los profesionales se combinan y son complementarias muchas de ellas. Según sus relatos, uno de los elementos claves es el conocimiento que van adquiriendo en el ejercicio, ya que este saber sintoniza a los profesionales con el mundo rural. Es importante mencionar que este conocimiento también permite la construcción de confianza entre profesional y usuario.

No es tan fácil, porque tú tienes que ir conociendo a la gente. La gente rural tiene una tendencia a conocer primero a la persona y una vez que... primero, te ponen distancia, se te desaparecen de los controles; después tú logras recuperar la adherencia a los controles, se empiezan a acostumbrar a ti; y recién ahí tú puedes empezar a hacer un trabajo más comunitario, recién ahí... y una vez que ya se han contado todos cómo es la situación y han compartido la experiencia de cómo se llevan contigo, ahí empiezan a aceptarte e incluirte

(matrona, Cecosf rural)

También surge de los discursos que los equipos utilizan el trabajo de terreno como una forma de insertarse en la comunidad, ya sea a través de la visita domiciliaria, la atención en domicilio, el rescate de pacientes, talleres comunitarios, entre otros.

Para un entrevistado, en este caso médico, un tema más técnico como la formación en salud familiar es la herramienta que les ha ayudado a insertarse con la comunidad, esta capacitación logró cambiar el enfoque inicial con el que llegó a trabajar en su comuna-isla.

Y relacionado a lo anterior, que también es parte del modelo, es el enfoque integral en la atención intra-box, lo que permite tener una mirada más sistémica de la persona la que es parte de una familia y un entorno.
Siempre fue trabajar con la familia ahí, siempre fue contar con... eh... con él, esta historia de la persona que se sentaba adelante y considerar todo y ver si podías... ver si el marido tenía problema ayudarla y empezar como sin querer queriendo metiéndote hacia la salud familiar. Yo creo que, por una cosa de tiempo, la manera de ser también, como que no a todos les gusta o les agrada, pero fue súper espontáneo, no hubo nada muy premeditado

(Matrona, Cecosf)

Para otros es el aporte que hace los profesionales del equipo de salud, quienes, desde su experiencia, inducen al profesional entrante en su llegada al territorio y de esta forma logran la inserción en la comunidad.

Fíjate que cuando yo llegué, yo lo logré básicamente a través del resto del equipo, fundamentalmente me fui incorporando al equipo y un poco a su trabajo. Lo que pasa es que este equipo tiene mucho conocimiento de trabajo rural y tiene la particularidad de que, yo diría un cincuenta por ciento del equipo es originario de Curaco de Vélez. Aparte yo ya venía con un conocimiento, no te olvides que yo nací y me crié acá; por lo tanto, la población la conocía en su mayoría, no un cien por ciento, pero tenía un conocimiento ya de mi infancia

(EU, Cesfam insular)

\section{Organización del profesional en la ruralidad}

Esta categoría aborda la organización operativa de los profesionales para dar atención diaria a los usuarios, incluye temas como: la ronda médica, resolutividad como principio y multiplicidad de funciones:

La base del trabajo rural es la realización de rondas médicas a Postas Rurales y Estaciones de Salud Rural, las que pueden ser sedes vecinales o construcciones comunitarias destinadas a recibir la atención que entrega la ronda médica. La frecuencia puede variar desde una ronda médica semanal hasta una mensual en los centros de donde provienen los entrevistados. En esta oportunidad se realiza atención profesional, en general para todos los usuarios que asisten a la ronda médica, sin rechazo en la atención por demanda espontánea. Otros centros estructuran más la atención, con agenda programada. Finalizada la atención en box, se realizan las visitas domiciliarias programadas por el técnico paramédico que está a cargo de la Posta de Salud. 
Los equipos entrevistados relatan que la política organizacional que ellos han adoptado es resolver todas las solicitudes del usuario que estén al alcance del establecimiento, en lo referente a derivación, formas de movilización, entre otros. Es lo que hemos denominado resolutividad como subcategoría de la organización de los profesionales.

Aquí a pesar de no ser un centro de urgencia, solamente centro de salud rural, como que aquí siempre se resuelve todo, y si hay un tema de especialidades que no se puede hacer acá, se les consiguen las horas, se les coordinan con los pasajes, entonces la gente se va como con todo casi listo y eso también a la gente le gusta mucho, no se le tramita, siempre se le trata de dar una solución, y si no se le puede resolver aquí, se le consigue hora en otro centro

(matrona, Cecosf)

Lo anterior, debido a que los profesionales están conscientes de la carencia de servicios básicos y conectividad de esas zonas, más aún en sectores insulares, entonces los centros realizan acciones que resuelven la mayor parte de los problemas asociados a salud de las personas.

Respecto a la multiplicidad de funciones los entrevistados señalan que, al ser únicos en sus establecimientos, deben organizar los insumos y procesos necesarios para la atención ya que no se cuenta con unidades de apoyo como en centros urbanos, por ejemplo, solicitud de insumos clínicos, de escritorio, esterilización, realización de estadística de atenciones, coordinación de horas a especialidad médica (mencionado anteriormente). También se deben encargar de gestionar con mucha anticipación los permisos legales, ya que los médicos, al ser únicos, requieren reemplazo en sus funciones.

Impacta la ausencia, por eso generalmente uno programa bien sus permisos, súper bien sus vacaciones, coordinando todas las atenciones, si yo no estoy yo ya sé que tengo que mandar una caja con anticonceptivos a la posta o dejar algo listo acá, uno les dice a las personas: si usted necesita algo, hable con los paramédicos

(matrona, Cecosf)

También como parte de la multiplicidad de funciones, el hecho de ser únicos en su profesión les demanda adquirir distintas jefaturas o responsabilidades. Dentro de los entrevistados, hay coordinadores del CECOSF, subdirectores de CESFAM y/o jefes de un programa, incluso algunos están a cargo de seis programas sanitarios en total.

\section{Situación de RHUS rural}

Ante la solicitud de describir la situación del recurso humano rural, los entrevistados mencionan la inestabilidad laboral que viven los equipos rurales, poseen contrato a plazo fijo y no tienen certeza sobre la renovación de ese contrato, que para algunos de ellos responde a temas políticos más que técnicos.

El siguiente relato constata la percepción sobre la institución que contrata al profesional y su relación con el posible impacto de la permanencia de éste.

Yo creo que el nivel de la salud de la población mejoraría mucho si aquí se empezara a cuidar al recurso humano. Aquí no se cuida al recurso humano. Nuestra Corporación no tiene un mayor interés en conservar el recurso; si bien nos proveen de capacitación, las condiciones laborales no son buenas y no son buenas porque no somos respetados, somos tratados como objetos

(matrona, 52 años, Cecosf)

Estos discursos tienen más bien relación con médicos municipales y profesionales matronas y enfermeras, ya que la modalidad de médicos EDFs asegura permanencia mínima de 3 y máxima de 6 años. Además, el año 2015 se amplió los cargos de médicos en etapa de destinación a comunas urbanas y rurales de la isla de Chiloé, lo que aumentó considerablemente el número de médicos en los lugares en donde existía brecha médica.

Al indagar sobre las percepciones de la situación del recurso humano rural en cuanto a permanencia, se pone el énfasis en la alta rotación de profesionales a diferencia de lo que ocurre con el estamento de técnicos y administrativos que se mantienen más estables debido a que la mayor parte de ellos estudiaron en la provincia de Chiloé o en provincias cercanas y además pertenecen al territorio donde está asentado el centro asistencial.

Hasta donde yo sé es alta rotación que hay de profesionales, por lo menos lo que es colegas, en estos 5 o 6 años ha habido 3 o 4 , conmigo 5 , cinco matronas que han ido y venido, la última fue mi colega que está ahora con licencia después de su posnatal que al parecer no va a venir, ella es de Antofagasta, bastante lejos y creo que ya no se devuelve. Entonces sí, la rotación es bastante alta

(matrona, Cecosf insular)

En este mismo sentido, algunos señalan que han 
vivido la experiencia de ausencia profesional prolongada, en donde la ocupación de ese cargo ha sido tardía e inconstante, sobre todo en los sectores más apartados. El impacto de esta ausencia se analiza en la siguiente categoría de análisis.

(...) las matronas, lo sé por el caso de la matrona que estuvo con su pre y pos natal el cargo a matrona estuvo abierto para reemplazo...no tuvimos ninguna matrona, reemplazaron algunas, no sé si supiste, de Castro, pero eran reemplazo por días y en extensión horaria. Después, no sé poh llegó una niña que era de Santiago, estuvo un mes, se aburrió, se fue

(médico EDF, Cesfam)

\section{Impacto de la permanencia}

Luego de analizada la situación actual del recurso humano, los entrevistados se refirieron al eventual impacto de la permanencia del recurso humano en el nivel de salud de la población. Las respuestas en general fueron en torno a la pérdida de la continuidad de atención que genera desconfianza entre los usuarios y el equipo profesional/sistema de salud, la ausencia de cobertura de prestaciones críticas de la cartera de servicios con y peores resultados sanitarios (en caso de ausencia profesional sin reposición del cargo en forma inmediata); y la postergación de las actividades preventivas y comunitarias para cubrir actividades de curación y rehabilitación en ausencia de profesional. Todo esto influye directamente en la calidad de atención a la población y así mismo lo relatan los entrevistados.

La continuidad de atención es mencionada como aspecto fundamental que se deteriora por la alta rotación y ausencia de profesionales. Lo que a su vez se relaciona con la afectación de la confianza en la relación médico-paciente, ante la incerteza de la permanencia del profesional de cabecera. Este vínculo que se genera en la relación médico-paciente es mas allá del rol profesional, si no con la persona tras ese profesional.

En este mismo aspecto, los entrevistados perciben que la no permanencia del RHUS hace que la población desconfíe del sistema de salud, un sistema que no asegura la presencia de sus funcionarios en el territorio y por ende el acceso a la salud.
Yo creo que impacta en un montón de niveles...desde la percepción que el usuario tiene del sistema de salud en general, hasta cómo él se siente viniendo o no al CECOSF cuando le cambian a su enfermera. O sea, en un nivel súper personal, hay gente... por ejemplo... nosotros hemos tenido rotación alta de enfermera y de psicólogo. Hay gente que estaba en terapia psicológica y que después no ha querido volver. Y no tiene nada en contra, viene a la matrona, viene a la doctora, pero al psicólogo no se acerca nunca más. Y otras personas que dicen que francamente para ellos es una desilusión del sistema de salud, del sistema en Chile porque se sienten, así como tan botados...

(matrona Cecosf)

En relación a lo anterior, algunos entrevistados reflexionan que el RHUS rural es el soporte del modelo de salud familiar, por lo tanto, los problemas de permanencia deterioran la instalación y continuidad del modelo.

Impacta fundamentalmente en la población, en la confianza, en el Modelo de Salud Familiar que busca que conozcas a tu población, que tú seas su médico de cabecera en el caso de los médicos, médico de la familia... no lo logras con una alta rotación de médicos

(EU, Cesfam insular)

Otro probable impacto sanitario de la permanencia mencionado por los entrevistados es la ausencia de cobertura de prestaciones críticas de la cartera de servicios. Esto, en el caso de la ausencia de un profesional en forma prolongada sin una solución oportuna de reemplazo, o cuando no se repone a tiempo el cargo luego de una renuncia, que en general se produce por falta de profesionales interesados. Algunos entrevistados consideran que las prestaciones de salud asociadas al programa de salud mental probablemente sean las más afectadas por la rotación de profesionales.

(Afecta) La calidad de salud de las personas indudablemente; porque si yo no tengo matrona voy a tener más embarazos, voy a tener más ingresos tardíos. Entonces de todas maneras que va a impactar en la salud de las personas

(EU, Cesfam insular)

La priorización de las actividades asistenciales y la postergación de las actividades preventivas y comunitarias para cubrir actividades de curación y 
rehabilitación en ausencia de profesional, es quizá una estrategia común para establecimientos de salud tanto urbanos como rurales. Sin embargo, cuando existe brecha profesional en un sector rural se evidencia más la postergación de estas actividades ya que el modelo de atención en la ruralidad se sustenta en acciones preventivas, comunitarias y en terreno, como base de la prestación de servicios

Como cierre del eje temático sostenibilidad de programas en la ruralidad decir que todas las particularidades de los servicios rurales descritas anteriormente son las que se afectan con la alta rotación y la no permanencia de profesionales. Estos aspectos generan una especificidad del trabajo rural y que es el que se ve mermado por la situación actual de permanencia del recurso humano rural.

\section{Eje Análisis de Políticas actuales de permanencia recurso humano rural}

En general existe un discurso con pocas certezas sobre la existencia de ellas, algunos profesionales no manejan información al respecto y otros consideran que no existen tales políticas. Algunos elementos que surgen son relacionados a incentivos económicos, el programa de apoyo de enfermería rural (PAER) y programa de destinación y formación de médicos (EDF).

Un grupo importante de entrevistados, todos ellos profesionales no médicos, respondieron que no existe una política ni una estrategia de permanencia de RHUS que beneficie a profesionales rurales. Es importante señalar que, en este punto, los profesionales no se sienten interpelados con las políticas de permanencia ya existentes, porque precisamente no son beneficiados por ellas.

Yo creo que no existe una política, porque si existiera yo creo que la hubiese visto reflejada en algún área en mí, porque llevo años trabajando en Chiloé en sectores rurales. Según yo creo que no existe, o si existe yo no la conozco o no la aplicaron en mí

(matrona Cesfam insular).

Los profesionales entrevistados no perciben ninguna estrategia de atracción, sólo mencionan la capacitación ofrecida por el Servicio de Salud para los establecimientos de atención primaria, pero luego precisan que esta es para todos los profesionales y no dirigidas exclusivamente a quienes se desempeñan en zonas rurales.

Respecto a políticas de retención específicamente, los discursos de los profesionales son más críticos aún, muchas respuestas de los entrevistados es considerar una ausencia total de ellas.

No. De hecho, lo que he visto es todo lo contrario. No te otorgan facilidades ni para el desplazamiento, ni facilidades... acá usamos el (box) multipropósito para almorzar, aquí hay lugares donde tú ni siquiera puedes comprar un agua mineral, donde no compras un pan... Entonces no te dan ninguna facilidad, como decir "ya, vamos a tener un lugar acogedor o adecuado para almorzar", por lo menos... no, no hay facilidades... "para el verano le vamos a traer un refrigerador para que traiga...", no, no hay... no se aprecia el mayor esfuerzo- como decía la pauta de calificación de mi otro lugar de trabajo- "no se aprecia el mayor esfuerzo" de la Corporación por mantener al recurso humano satisfecho.

(Matrona Cecosf)

Por otro lado, los incentivos económicos se mencionan como estrategia de atracción, a saber: bono zonas extremas trimestral, asignación de desempeño difícil y viáticos por desplazarse a rondas médicas. Sin embargo, para los profesionales el incentivo de la asignación por desempeño difícil no es suficiente para asegurar que los profesionales permanezcan en un sector apartado, es decir, no se logra la retención la que depende de otros factores que pueden ser externos al establecimiento como la geografía o la insularidad con todas las implicancias de acceso que eso conlleva (salud y educación, por ejemplo)

La única forma de atracción que tienen es el hecho de que en algunas partes, no en todas, te pagan desempeño difícil, pero eso no hace una diferencia ostensible con el sueldo que tú podrías recibir en Santiago.

(Matrona, Cecosf)

La estrategia del programa de apoyo a la enfermería rural, es mencionada como una forma de atraer y mantener a enfermeras, sin embargo, debido a lo escasamente señalada por los entrevistados se infiere que esta medida no ha sido lo suficientemente posicionada. 
Con las enfermeras creo que hubo un tiempo la posibilidad de que hicieran eso (PAER), ya, pero no sé si se mantiene, yo no tengo la claridad de eso, pero creo que todo el resto de los profesionales tipo B o profesionales no médicos también deberíamos tener la posibilidad de tener esa opción, pero esa sería una buena política

(matrona, Cesfam insular)

A diferencia del anterior, el programa de médicos en Etapa de Destinación y Formación (artículo 8, ley 20.664) aparece en los relatos de los entrevistados como una política de mayor consolidación entre los médicos y es de conocimiento de todos los profesionales entrevistados, la que es considerada como política de atracción y retención a la vez.

Respecto a políticas o estrategias locales de permanencia los entrevistados mencionan una asignación municipal que una comuna paga a sus funcionarios (de donde provenían entrevistados), sin embargo, ésta es indiferenciada, beneficia a profesionales tanto del sector urbano como rural. En dos CECOSF insulares que cuentan con equipo profesional, se les paga un bono de pensión o se les otorga vivienda para residir en el mismo territorio, este beneficio no está dirigido a todos los profesionales, sino a los que conforman el equipo básico. Otra medida local, mencionada por un profesional es la existencia de dos días de permiso mensuales llamado "días de pago", el cual es discrecional del empleador y no está normado según lo señalado en el siguiente relato:

En el análisis local de estrategias, surge un elemento interesante de mencionar, no considerado como política pero que está presente en un establecimiento y que sin duda incide en la retención de profesionales. Tiene relación con el clima laboral que la jefatura ha fomentado, según la entrevistada, promoviendo en forma tácita el buen trato y calidez entre los compañeros de trabajo, elemento que se vincula también con las motivaciones de los profesionales analizados en su correspondiente eje.

Factores y escenario actual de políticas de permanencia

Luego de realizar el diagnóstico de las políticas actuales de permanencia de RHUS rural se identifican las razones que dan lugar al déficit de éstas o a la forma en que han sido abordadas, a saber: evaluación poco pertinente y limitada de la atención en salud rural (cuantitativa), centralización de la gestión en salud, comunidad no organizada y equipo profesional poco demandante que no releva su quehacer.

Los profesionales coinciden en que la forma de medir la atención en salud rural, tanto en niveles regionales como centrales no es pertinente a las acciones diferenciadas que se dan en este contexto. Refieren que la evaluación es sólo cuantitativa, se compara con la urbana en términos absolutos y no considera elementos cualitativos ni las particularidades de la atención rural, lo que hace que las necesidades de los equipos no sean abordadas en su contexto real.

Entonces te preguntan si estás atendiendo a tal porcentaje de la población, cuánto porcentaje de la población compensaste, pero hay un tema cualitativo que se deja de lado y que yo no sé si el ministerio sabe, ¿sabe que existen esos problemas?

(matrona, Cecosf)

Relacionado con lo anterior, mencionan el desconocimiento de las autoridades sobre la ejecución del trabajo en un sector rural, lo que se traduce también en una evaluación poco pertinente de los establecimientos rurales.

Por una simple y sencilla razón, porque la gente que está en los puestos de mando y porque nuestros propios colegas que trabajan en el resto de la comuna, no tienen la más remota idea de lo que es trabajar en rural. Porque ellos piensan todos que tú llegas a una oficina; que tú no tienes mayores dificultades de traslado, "si tienen un bus", ¿y si el bus queda en pana?, ¿cómo llegas tú?

(matrona, Cecosf)

Emerge también de los relatos el concepto de centralización en salud, que se materializa en el hecho de que las autoridades en salud centren su atención y recursos a los problemas de los sectores de mayor densidad poblacional. Esta mirada de postergación también fue analizada en el eje temático de necesidades, desde el enfoque de aislamiento, sufrido por el equipo y la comunidad misma.

Reconocen también como factor condicionante el bajo empoderamiento de la comunidad y profesionales, en donde la participación de la comunidad, en la exigencia de derechos es aún inicial, según las percepciones de los entrevistados y eso hace que las autoridades no logren percibir sus necesidades en salud. 
Creo que otro factor importante es que usuarios todavía, si bien están aprendiendo a alzar la voz y decir lo que necesitan, creo que la participación comunitaria todavía es débil, que, si la gente exigiera cosas, lograría muchas más cosas que si nosotros hablamos, la gente tiene mucho poder, lo que pasó en Quellón es reflejo de aquello

(Matrona, Cesfam insular)

Y por otro lado reconocen la pasividad que presentan como equipo en la exigencia de condiciones básicas para trabajar junto con el poco énfasis que realizan de su labor en las zonas apartadas. Estas razones, según los entrevistados, condicionan la ausencia de políticas de permanencia pertinentes a sus necesidades.

El diagnóstico de políticas actuales de permanencia de recurso humano rural, diferenciado en los grupos se analiza de la siguiente forma: el discurso de los médicos es en torno al programa de destinación y formación como exclusivo para ellos y a la falta de incentivos para la retención. En el caso de matronas y enfermeras, los relatos son sobre ausencia de políticas de retención igualmente, excepto el Programa de Apoyo a la enfermería rural, visto como única estrategia por algunas entrevistadas, en franca disminución por otras y desconocida por las entrevistadas más jóvenes. Para las matronas no existe política de permanencia específica, lo cual es visibilizado por este grupo de profesionales.

\section{Eje Construcción de Política de per- manencia recurso humano rural desde los actores}

Las propuestas que realizan los profesionales son en distintos ámbitos, desde la especialización profesional, la formación continua, el mejoramiento de las condiciones de vida y laborales tanto en términos contractuales como condiciones físicas.

Estrategia de formación en pregrado: formación a estudiantes en salud rural y pasantías obligatorias a estudiantes de profesiones sanitarias en establecimientos rurales y apartados como parte del currículo. También se menciona la captación de estudiantes que cuenten con perfil para trabajar en zonas rurales, como por ejemplo aquellos que provienen de estos territorios.

Estrategias de formación a profesionales: capacitación para profesionales rurales, diferenciada y continua, las matronas entrevistadas proponen un Programa de destinación y formación para matronas, similar a la modalidad enfermeras PAER y médicos EDF. Los médicos municipales sugieren una estrategia más expedita para acceder a especialidad médica, que sea específica para quienes se desempeñan en sector rural. Los médicos EDF proponen reinstalar el beneficio de la capacitación "mes por año" que en algún momento existía para los médicos, en donde ellos en forma individual gestionaban pasantías con especialistas de hospitales.

Algunos consideran que la falta de Universidad en la zona limita el acceso a capacitación y proponen alianzas con casas de estudios para la realización de planes anuales de formación a profesionales de salud en el mismo territorio.

Estrategias de incentivos económicos: los profesionales no médicos sugieren mejoras en remuneración, bono de pensión, bono de movilización, cobertura de gastos de instalación y mudanza como medidas posibles de aplicar. Por otro lado, se señala la implementación de un bono de permanencia, es decir, que al cumplir una cantidad de tiempo específico se acceda a este bono especial de retención.

Estrategia de mejoras en condiciones contractuales: proponen estabilidad laboral para mejorar la permanencia de los profesionales y eliminar el contrato de honorarios para los funcionarios que se desempeñen en zona rural. Además, aquellos que se desempeñan en sectores insulares proponen plan de seguridad y capacitación en salvataje.

Estrategias de bienestar de profesional: En caso de que el empleador disponga de viviendas para el equipo rural, que estas sean adecuadas y acorde al número de los profesionales destinado en ese sector rural. También algunos profesionales mencionan como estrategia la implementación de planes de autocuidado para el personal, especialmente por el requerimiento físico asociado al trabajo en terreno, largas caminatas, carga constante de maletas y cajas para la realización de rondas rurales, entre otras actividades que demandan esfuerzo físico. Por otro lado, una profesional entrevistada señala la disposición de permisos legales diferenciados para estar con la familia, para profesionales que residan en zonas apartadas. Además, los entrevistados consideran necesarias estrategias de reconocimiento desde las jefaturas a los profesionales cuando el desempeño de éstos es destacado, a través de mayores responsabilidades dentro del equipo.

En sentido estructural, también se menciona la idea de que las autoridades mejoren el acceso al sector rural: caminos, rampas y sistema de transporte público.

Cierre de brecha de infraestructura de los establecimientos rurales y condiciones mínimas para el desempeño rural: calefacción, acceso a internet, 
teléfono, insumos generales y clínicos adecuados.

Soporte profesional y personal: Mejorar resolutividad a nivel local, otorgar más complejidad a la atención, por ejemplo: telemedicina, consultorías especialistas en terreno y rehabilitación con base comunitaria, para así generar redes de profesionales y evitar el aislamiento profesional. Por otro lado, los entrevistados mencionan la necesidad de realizar jornadas de encuentro entre otros equipos rurales para mejorar el trabajo en red.

Estrategia basada en la pertinencia de la evaluación y de políticas de salud rural: proponen una evaluación pertinente y diferenciada para la atención rural, que las autoridades o jefaturas sintonicen con las necesidades del personal a través de un flujo de información expedito desde equipos rurales a tomadores de decisiones, para así lograr políticas que tengan coherencia con las necesidades reales de los profesionales.

Las propuestas de políticas de permanencia para el grupo de médicos son relacionadas principalmente con la especialización, por un lado, el grupo de médicos municipales propone estrategias que faciliten el acceso a programas de residencia, por otro los médicos EDF indican mejoras en los beneficios de capacitación "mes por año" que está en retirada en los servicios de salud. Otra propuesta de este grupo es que la evaluación y medición que se realiza a los equipos rurales sea diferenciado y pertinente, ya que en general la comparación es con la población y productividad de los equipos urbanos. También señalan la necesidad de contar con estrategias de resolutividad incluidas en la propuesta de soporte profesional y personal, con el fin de hacer redes con especialistas del nivel secundario de atención y generar mayor complejidad a su labor en atención primaria.

El grupo de matronas y enfermeras proponen principalmente mejoras en las condiciones económicas, implementación de un sistema de especialización asociada a permanencia como el caso de los médicos EDF y que exista una política que exija el cumplimiento de las condiciones mínimas de instalaciones, insumos clínicos, servicios generales, entre otras. Esa última propuesta es compartida por ambos grupos, junto con la capacitación continua y mejoras estructurales en el acceso a los territorios (conectividad, rampas, movilización).

\section{Discusión}

Se reconoce que pese a los esfuerzos de algunos países, aún persiste el problema de la distribución inequitativa del RHUS, con alta concentración en las zonas urbanas lo que produce problemas de retención en zonas rurales (Organización Panamericana de la Salud y Organización Mundial de la Salud, 2015).

Esta premisa dio origen a la realización de esta investigación cuyos principales resultados se vinculan con las motivaciones de los profesionales para trabajar en un sector rural, las necesidades sentidas por los profesionales las cuales difieren de acuerdo al grupo (profesión y momento laboral) y las propuestas para fortalecer la retención de éstos. El discurso de los entrevistados estuvo enmarcado en la carencia de políticas de estado que promuevan la permanencia de profesionales en zonas apartadas.

De acuerdo a los hallazgos del estudio, para el grupo de profesionales matronas y enfermeras, su motivación principal es el vínculo familiar en el territorio y disfrutar de las condiciones de la vida rural, entorno e idiosincrasia. El factor económico es transversal a todas estas motivaciones, pero no suficiente por sí solo. En las profesionales más jóvenes se agrega la motivación asociada al constante desafío que les significa trabajar en sectores rurales, ya que profesionales recién egresados ponen a prueba sus conocimientos y habilidades recién adquiridas en el pregrado. Los resultados de esta tesis aportan información acerca de las motivaciones para este grupo de profesionales de los cuales no existía evidencia hasta ahora en nuestro país.

Sin duda, el trabajo en las zonas rurales presenta particularidades que los entrevistados mencionan como relevantes para entender el contexto en que se prestan los servicios, se vinculan con las motivaciones de los mismos y generan también necesidades, a saber: problemas de acceso tanto del usuario al centro como del profesional hacia el usuario, dinámica de la atención más flexible en cuanto a la agenda de atención, cercanía en la relación profesional-usuario marcado por la empatía y respeto a la cultura; y el rol social del profesional rural en su comunidad. En este último punto, el rol social ejercido por el profesional como integrante de la comunidad, lo convierte en un actor relevante que asume liderazgo y gestiones en otras áreas de la salud esenciales en un sector rural, por ejemplo, servicios sanitarios básicos como alcantarillado, agua potable, disposición de basura, problemas de impacto ambiental, etc. Esto, debido a que en el sector rural no existen otros profesionales dedicados a estos temas, y si existen, no tienen contacto directo con la comunidad como sí lo tiene el equipo de salud que se asienta en el territorio. Una reflexión interesante que surge a partir de estos resultados es la concepción de salud de la comunidad rural la que es vista de una forma más integral y menos reducida a lo meramente asis- 
tencial, como podría ocurrir en un sector urbano. Aquí cobra relevancia el enfoque de determinantes sociales en salud (Organización Mundial de la Salud, 2011), que implícitamente se configura en la comunidad y también en el equipo, en el contexto de una zona rural con características y particularidades que propician la implementación integral del Modelo de Atención con enfoque familiar y dentro de este un rol comunitario más preponderante.

Estos elementos diferenciadores requieren de un soporte profesional, es decir equipos presentes en los territorios, que se integren a la comunidad y que permanezcan en ella. Precisamente estas particularidades que caracterizan y nutren la salud rural son las que se ven afectadas por los problemas de permanencia del RHUS, junto a un modelo de atención integral y familiar que pierde continuidad y credibilidad.

Este contexto de la atención lleva a una forma de organización del profesional caracterizado por trabajo en terreno (ronda médica, visitas domiciliarias) y multiplicidad de tareas a desarrollar, clínicas, administrativas y de gestión. Esta mayor responsabilidad que les toca asumir, se da en condiciones de aislamiento profesional, en un contexto de grandes distancias, problemas de acceso, dificultades para desplazarse, con sistemas de comunicación deficitarios, entre otras circunstancias que dificultan el quehacer sanitario y los diferencia ampliamente de los colegas que ejercen la misma labor en un sector urbano. Estos resultados, surgidos de las vivencias de los profesionales van en la misma línea de los resultados de otros estudios que han comparado los servicios sanitarios de sectores rurales y urbanos. De igual modo, ya ha sido descrito en la evaluación que hace la OMS del escenario de los RHUS rural, el que se caracteriza por falta de recursos y en donde "muchas veces la administración de estos centros recae en los mismo profesionales aumentando los niveles de responsabilidad y eventualmente de frustración" (Organización Panamericana de la Salud y Organización Mundial de la Salud, 2015; Smith et al., 2008).

En relación a lo anterior, la situación del recurso humano rural en salud, según los entrevistados, es caracterizada por inestabilidad laboral, alta rotación (excepto los técnicos en salud) y cargos no cubiertos que genera barreras de acceso a la atención profesional. Este diagnóstico no es distinto en otras regiones de las Américas, y que es lo que ha dado sustento a la creación de recomendaciones de fidelización de RHUS por la OMS y la implementación de algunas estrategias en los países que han adscrito a estos acuerdos (Dolea et al., 2009).

De acuerdo a los entrevistados, uno de los impac- tos de la no permanencia está dado por la ausencia de prestaciones críticas que se traducen en peores resultados para la salud de la población. Los profesionales suponen y ejemplifican que las ausencias de profesionales sin reposición inmediata, afecta la supervisión del embarazo como atención prioritaria, en el caso de Programa de la Mujer. En este punto, es interesante mencionar que indicadores como la mortalidad materno-infantil mejoran en la medida que los países presentan mayor densidad de RHUS calificado (médicos, enfermeras y matronas) (Organización Panamericana de la Salud y Organización Mundial de la Salud, 2015).

Dentro de las políticas o estrategias actuales, sin duda la Etapa de Destinación y Formación para médicos es la que ha tenido impacto en la permanencia de médicos en las zonas más apartadas y en el aseguramiento del acceso a la salud de los habitantes de esos territorios. Esta política ha cumplido su objetivo que se refleja en un $100 \%$ de plazas ocupadas a lo largo del país, por lo mismo Chile declara para el periodo 2014-2018 la inyección de 210 nuevas plazas para médicos en zonas rurales o aisladas, con el fin de mejorar coberturas en las comunas más desatendidas y con índices de desarrollo humano más bajos (Organización Panamericana de la Salud y Organización Mundial de la Salud, 2015). Esta política se constituye en una motivación mencionada por los profesionales médicos para ocupar las plazas de las zonas rurales, que ha permanecido y se ha consolidado a través del tiempo.

En la evaluación de los países de la región andina realizada el año 2015 en relación a esquemas de incentivos para la retención, la crítica a Chile es que en el ámbito de las profesiones no médicas no existe un set de incentivos o una intervención específica para desempeñarse en zonas rurales, excepto ciertas compensaciones económicas en comparación a puestos de trabajo en zonas urbanas (Organización Panamericana de la Salud y Organización Mundial de la Salud, 2015). La mayor parte de los entrevistados perciben que no existe política de permanencia que los beneficie y consideran que los incentivos económicos (bono zonas extremas trimestral y asignación por desempeño difícil) no compensan el esfuerzo, las condiciones precarias y riesgos a los que se exponen en el ejercicio de su labor. En efecto concluyen que estas estrategias no promueven su retención en el territorio, la que más bien se produce debido al compromiso de los profesionales y a la existencia de un vínculo familiar o social que los hace permanecer más tiempo. Al realizar esta reflexión ellos expresan su necesidad de una política de atracción y retención de RHUS teniendo como referente el programa de médicos EDF ya que el programa de Enfermería Rural, como experiencia piloto, no ha tenido continuidad en el tiempo ni 
suficiente posicionamiento y sólo se menciona por algunos entrevistados como una estrategia no consolidada (Sandoval, 2002).

Un aspecto discutido de forma enfática por gran parte de los profesionales es la evaluación limitada de las autoridades, la centralización como parte de este abordaje poco pertinente y la constante comparación de población, productividad y procesos con los establecimientos urbanos, situación que no les favorece como equipos rurales. Los entrevistados proponen que las evaluaciones consideren la necesidad y diferenciación de los servicios rurales, ya que un enfoque exclusivamente cuantitativo, no da cuenta de los requerimientos reales de la población. Por ejemplo, a la hora de determinar la cantidad de profesionales por territorio, la OMS señala que las necesidades de salud de las poblaciones rurales son mayores por lo que será necesario un número proporcionalmente más alto de agentes de salud en esas zonas. Lo anterior, sin desconocer que el número de población es menor en una zona rural, sin embargo, los requerimientos de esa población son mayores, y en ese sentido las comunidades pequeñas de zonas remotas pueden requerir un número más elevado de médicos generales en relación a densidad poblacional que el que se justificaría en un contexto urbano. Este aspecto se vincula además con la implementación del modelo de salud considerando un eje comunitario importante, lo que requerirá de profesionales a cargo de menor población, en un escenario rural más disperso y con dificultades de acceso. (Organización Mundial de la Salud, 2011).

Es preciso señalar que las condiciones inadecuadas para el desempeño profesional en un contexto geográfico difícil son comunes en todos los entrevistados, pero la tolerancia y adaptación a ellas, sumado al compromiso con el trabajo rural hacen que los profesionales decidan permanecer en el territorio en mayor o menor medida- pese a la precariedad de las condiciones de laborales. La retención se torna frágil -y no garantiza un acceso sanitario para la población rural que requiere de los servicios en un contexto de vulnerabilidad y postergación- cuando está más supeditada a motivaciones personales e individuales que al éxito o fracaso de una política pública. En este sentido y tal como ha mostrado los resultados de esta tesis, el establecimiento de una política de atracción y retención para el equipo profesional básico que considere las necesidades y motivaciones de estos, es fundamental para asegurar cobertura y continuidad de atención de salud para la población rural.

\section{Conclusiones}

Esta investigación responde a la interrogante de cuáles son las motivaciones a la permanencia de los distintos profesionales que se desempeña en zonas rurales y cuáles son sus percepciones acerca de las políticas de retención. Los factores que inciden en la atracción y retención del RHUS rural son aquellos vinculados con las motivaciones, expectativas y necesidades de los profesionales en un contexto de particularidades del sector rural que moldean su ejercicio profesional. Aquí las condiciones de vida incluidas el aislamiento, las condicionantes para el desempeño laboral, la escasa valoración actual de los equipos rurales y una evaluación poco pertinente de los servicios rurales son vistas como necesidades y a la vez explicaciones de los profesionales hacia la política deficitaria de permanencia de RHUS en Chile. Los hallazgos sobre motivaciones coinciden en gran parte con lo planteado por la OMS y la mayor diferencia en los dos grupos estudiados es la relacionada con el incentivo a la especialización en el grupo de médicos.

Este estudio exploratorio, genera información relevante sobre motivaciones, necesidades y propuestas de profesionales no médicos, ausente hasta ahora en Chile, ya que los estudios existentes han surgido del programa de médicos EDF como único paquete de incentivos que beneficia al recurso humano rural (Peña et al., 2010). Los resultados muestran la importancia de estimar la acción conjunta del equipo básico de salud a través de generar un programa de incentivos para los profesionales médicos y otros profesionales demostrando una valoración del equipo en su conjunto. Es preciso especificar que los relatos analizados corresponden a profesionales de salud que se desempeñan en zonas rurales de Chiloé, que si bien aporta información relevante en este tema no necesariamente representa a aquellos profesionales que se desempeñan en otras zonas rurales del país con distintas condiciones geográficas.

Junto con lo anterior y entendiendo que para la aplicación del modelo de salud familiar se necesita de un equipo de salud interdisciplinario, es preciso señalar que en zonas rurales el modelo tendría un mejor desempeño y mayor posibilidad de implementación debido a su contexto, el que se caracteriza por sus particularidades: atención integral y cercana, la vinculación y el rol social del profesional con la comunidad. Y justamente son estas especificidades de la salud rural las que se ven afectadas con la no permanencia o la alta rotación del recurso humano junto con otros aspectos mencionados por los profesionales y que son claves para los servicios sanitarios rurales: continuidad de la atención, integración del profesional en el territorio, cumpli- 
miento del modelo de salud familiar y confianza en la relación profesional-paciente.

La respuesta a estas necesidades supone entender las distintas ruralidades existentes en Chile, que la institucionalidad aborde la salud rural como una unidad diferenciada dentro de la atención primaria y que los criterios de evaluación de ambos sean distintos en su forma, para así evitar que los equipos rurales se sientan postergados o en competencia con establecimientos urbanos a la hora de resolver sus demandas.

Por otro lado, el diseño e implementación de la política requiere de una inversión a largo plazo y de un enfoque más allá del sector salud, una política intersectorial en donde distintos ministerios estén convocados a participar y los esquemas de incentivos sean relevados como políticas de Estado. Las propuestas realizadas por los profesionales son: pasantías obligatorias en pregrado, universidades cercanas a territorios, programas de destinación-formación para médico, matronas y enfermeras, incentivos económicos, mejores condiciones de contrato, viviendas adecuadas, mejorar accesos (rampas, caminos, transporte), mejores condiciones de infraestructura laboral, aplicación de tecnologías de soporte profesional y evaluación pertinente a los establecimientos rurales. En estas estrategias sugeridas se necesita la articulación de varios sectores del estado, tanto en aspectos financieros, de gestión y de inversión estructural, es decir, requiere ser abordado bajo el enfoque de salud en todas las políticas (Organización Panamericana de la Salud y Organización Mundial de la Salud, 2014).

Las estrategias estructurales que proponen los profesionales como equipamiento comunitario, transporte, acceso adecuado e instituciones educacionales en el territorio, si bien fortalecen la permanencia del recurso humano, al mejorar las condiciones de vida, también favorecería a la comunidad rural en su conjunto. En este sentido, el enfoque de determinantes sociales en salud nos da una forma de abordaje a la problemática rural, en donde el profesional de salud rural es parte de una misma comunidad junto a los habitantes y son ambos potenciales beneficiarios de una misma política de estado.

En síntesis, este estudio ha mostrado que los profesionales entrevistados que trabajan en el sector rural, pese a las condiciones precarias de trabajo (de todos) y de vida (de algunos), se mantienen en sus lugares de trabajo, con necesidad de incentivos adecuados, pero con alto compromiso por la población que tienen a cargo, especialmente en el grupo de matronas y enfermeras. Sin embargo, la permanencia se torna frágil, ya que son los factores indi- viduales como el vínculo familiar, la etapa del ciclo vital y el compromiso, los que finalmente generan la permanencia. Debido a esto, es justo y necesario diseñar e implementar un set de incentivos financieros y no financieros, abordar las condiciones de trabajo y soporte de tecnologías como parte de una política de atracción y retención, no sólo dirigida a médicos, sino a otros profesionales no médicos que conforman el equipo básico de salud.

Chile se caracteriza por poseer sectores aislados como parte de la geografía, y los habitantes de esas zonas siempre necesitarán acceso a la salud en forma oportuna, independiente de los vaivenes del mercado laboral del personal sanitario. Este escenario requiere de una respuesta del Estado, a través de una política de permanencia de recurso humano rural pertinente, con inversión a largo plazo y participación intersectorial, en el marco del principio de equidad sanitaria.

\section{Referencias}

Dolea, C., Stormont, L., y Braichet, J.-M. (2010). Evaluated strategies to increase attraction and retention of health workers in remote and rural areas. Bulletin of the World Health Organization, 88(5):379-385.

Dolea, C., Stormont, L., Shaw, D., Zurn, P., y Braichet, J. M. (2009). Increasing access to health workers in remote and rural areas through improved retention. Background paper for the first expert consultations on developing global recommendations on increasing access to health workers in remote and rural areas through improved retention. 2009. Geneva, WHO.

Ministerio de Salud (1991). Reglamento de Becarios de la Ley $N \hat{A}^{\circ}$ 15.076. República de Chile, Santiago de Chile.

Organización Mundial de la Salud (2011). Declaración política de Río sobre determinantes sociales de la salud. En Conferencia Mundial sobre determinantes sociales de la salud. Organización Mundial de la Salud.

Organización Panamericana de la Salud (2012). Health in the Americas: 2012 Edition. Regional Outlook and Country Profiles. PAHO, Washington D.C.

Organización Panamericana de la Salud y Organización Mundial de la Salud (2013). Medición y Monitoreo de Metas Regionales en Recursos Humanos en Salud. 
Organización Panamericana de la Salud y Organización Mundial de la Salud (2014). Plan de Acción sobre la Salud en todas las políticas.

Organización Panamericana de la Salud y Organización Mundial de la Salud (2015). Desarrollo de Esquemas de Incentivos para la Retención - Fidelización de Personal de Salud en Áreas Rurales y de Difícil Acceso. Serie Estudios Multipaís, 5:161 .

Peña, S., Ramirez, J., Becerra, C., Carabantes, J., y Arteaga, O. (2010). The Chilean Rural Practitioner Programme: a multidimensional strategy to attract and retain doctors in rural areas. Bulletin of the World Health Organization, 88(5):371-378.

Sandoval, C. (2002). Investigación cualitativa. Programa de Especialización en teoría, métodos y técnicas de investigación social. Bogotá, Colombia: Instituto colombiano para el fomento de la educación superior.

Smith, K. B., Humphreys, J. S., y Wilson, M. G. A. (2008). Addressing the health disadvantage of rural populations: how does epidemiological evidence inform rural health policies and research? Australian Journal of Rural Health, 16(2):56-66. 